\title{
Observation and investigation of plasimid DNA using an atomic force microscope
}

\author{
Yuan-Ron $\mathrm{Ma}^{1}$, H.-J. Chiang ${ }^{2}$, and Yung-Der $\mathrm{Yao}^{3}$ \\ ${ }^{1}$ Institute of Biotechnology, National Dong Hwa University, Hualien 974, Taiwan, \\ R.O.C. \\ ${ }^{2}$ Department of Physics, National Dong Hwa University, Hualien 974, Taiwan, R.O.C. \\ ${ }^{3}$ Institute of Physics, Academia Sinica, Taipei 115, Taiwan, R.O.C.
}

By mean of an ambient atomic force microscope 22-kb-pMT1 plasmid DNAs are observed in the tapping mode. A perfect circle-shape compact conformation is presented at the first time.

\section{Introduction}

Relaxed and closed-circular 22kb-pMT1-plasmid DNAs were observed by means of an ambient atomic force microscope (AFM) in the tapping mode (TM). Most of plasmid DNAs were reconstructed into two compact conformations on a mica surface, which are a normal relaxed compact structure and a new perfect circle-shape compact structure. This perfect circle-shape compact plasmid DNAs is observed at the first time. In this paper the perfect circle-shape compact plasmid DNA is presented and compared with the normal relaxed compact DNA.

\section{Experiments}

A 10-microliter binding-buffer solution was dropped on a mica, and then the other 10-microliter plasmid DNA was dropped to join the binding-buffer solution for one minute. Then the surface was rinsed with de-ion water several times and dried with $\mathrm{N}_{2}$ gas. The DNAs are pMT1 plasmid and have the size of $22 \mathrm{~kb}$ (conc.: 25 nano-gram per micro-liter). The solution of the binding buffer contains $10 \mathrm{mM}$ HEPES and $5 \mathrm{mM}$ $\mathrm{MgCl}_{2}$, and its $\mathrm{pH}$ value is 7.5.

\section{Results and discussion}

In Figure 1 an AFM image obtained in the tapping mode (TM) shows two closed-circular compact conformations of 22-kb-pMT1-plasmid DNAs. They are a normal relaxed structure and a perfect circle-shape compact structure, respectively. The large perfect circle-shape compact structure is observed at the first time. 


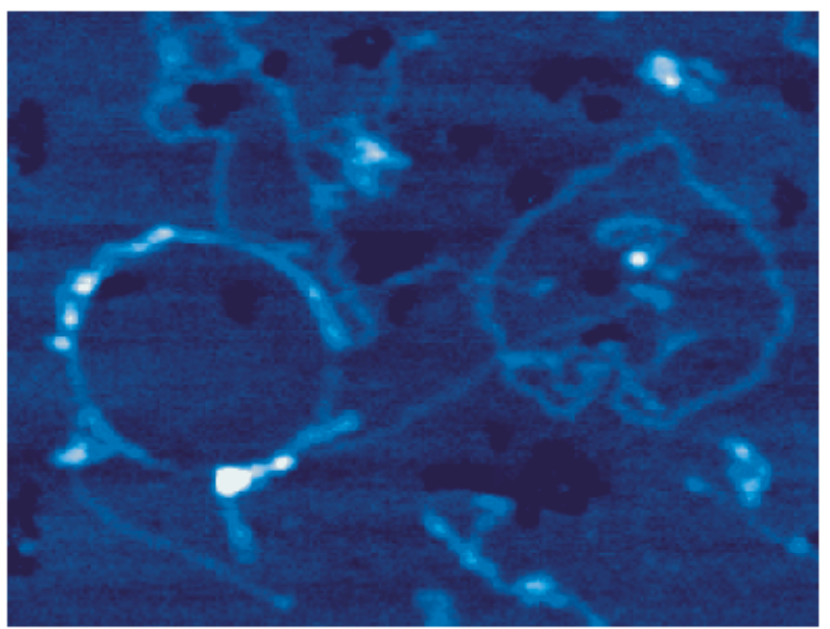

FIG. 1. AFM image $\left(1,700 \times 1,300 \mathrm{~nm}^{2}\right)$ showing two conformations of 22kb-pMT1-plasmid DNAs: the normal relaxed compact structure (right) and perfect circle-shape compact structure (left).

According to the DNA folding model of Martinkina et al. [1], schematic diagrams in Fig. 2 (a) and (b) show two possible models of the triplex DNA folding conformations for the perfect circle-shape and normal relaxed compact structures. The large DNA folding may be due to the water-sample interaction on the mica surface.

(a)

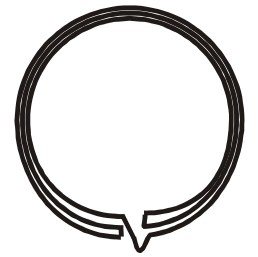

(b)

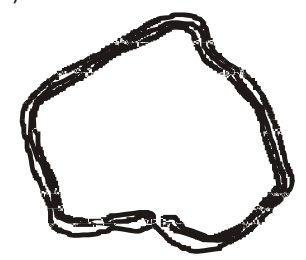

FIG. 2. Two models of the triplex DNA folding conformations.

\section{Conclusion}

A perfect circle-shape compact conformation of $22 \mathrm{~kb}$-plasmd DNAs is presented at the first time. The water-sample interaction suggested results in the model of DNA folding.

\section{References}

[1] Martinkina et al. J. Biomol. Struct. \& Dynam. 17 (2000), 687. 